\title{
Long-term clinical outcome of coronary artery stenting or coronary artery bypass grafting in patients with multiple-vessel disease
}

Shun Kohsaka, MD, ${ }^{\text {a,d }}$ Masashi Goto, MD, MPH, ${ }^{\text {e }}$ Salim Virani, MD, ${ }^{\text {a,d }}$ Vei-Vei Lee, MS, ${ }^{\text {b }}$ Noriaki Aoki, MD, PhD, ${ }^{\text {e }}$ MacArthur A. Elayda, MD, PhD, ${ }^{a, b}$ Ross M. Reul, MD, ${ }^{c}$ and James M. Wilson, MD ${ }^{a, d}$

From the Division of Cardiology, ${ }^{\mathrm{a}}$ Biostatistics, ${ }^{\mathrm{b}}$ Cardiovascular Surgery, ${ }^{\mathrm{c}}$ the Texas Heart Institute at St Luke's Episcopal Hospital, the Divisions of Cardiology and Cardiovascular Surgery, Baylor College of Medicine, ${ }^{\mathrm{d}}$ School of Health Information Sciences, the University of Texas at Houston, ${ }^{\mathrm{e}}$ Houston, Tex.

Received for publication July 11, 2007; revisions received Oct 26, 2007; accepted for publication Nov 1, 2007.

Address for reprints: Shun Kohsaka, MD, Division of Cardiology, $622 \mathrm{~W}$ 168th St PH 3-137, New York, NY 10032 (E-mail: sk2798@columbia.edu).

J Thorac Cardiovasc Surg 2008;136:500-6 0022-5223/\$34.00

Copyright $\odot 2008$ by The American Association for Thoracic Surgery

doi:10.1016/j.jtcvs.2007.11.050
Objective: Recent large-scale observational studies have shown better outcomes after coronary artery bypass grafting than after angioplasty or stenting in patients with multiple-vessel disease. The time frames of these studies, however, include periods of varying behavior with respect to patient selection, stent technique and design, and medical therapy. Our objective was to examine long-term outcomes of coronary stenting and coronary artery bypass grafting, including those performed in the contemporary era of aggressive medical therapy.

Methods: We examined in-hospital and long-term follow-up data from consecutive patients with multivessel coronary artery disease who underwent isolated initial revascularization by coronary stenting or coronary artery bypass grafting between 1995 and 2003. Cox proportional hazards modeling with propensity scoring and propensitybased case matching were used to compare long-term survival and correct for baseline differences between the populations.

Results: A total of 6847 patients were studied (stenting 3917, coronary artery bypass grafting 2930). Each patient had 1 to 9 years of follow-up (median 3.5 years). Unadjusted long-term mortalities were similar for coronary artery bypass grafting and stenting (hazard ratio $1.1,95 \%$ confidence interval $0.9-1.2, P=.21$ ). Matched comparison of 3488 patients (1856 in each group) with similar likelihoods of undergoing coronary stenting or coronary artery bypass grafting, however, suggested that coronary artery bypass grafting provided better long-term survival (hazard ratio $0.7,95 \%$ confidence interval $0.6-0.9 ; P=.004)$.

Conclusion: During a 9-year period, in physician-selected patients with favorable demographic characteristics for both revascularization procedures, coronary artery bypass grafting was associated with better long-term survival than stent-assisted angioplasty.

$\mathrm{R}$ ecent large-scale observational studies have reported better outcomes with coronary artery bypass grafting (CABG) than with coronary stenting in patients with multivessel coronary artery disease (CAD) ${ }^{1,2}$ The reported adjusted hazard ratios [HRs] for the long-term risk of death in these two studies ranged from 0.43 to 0.76 for patients who underwent $\mathrm{CABG}$ relative to patients who underwent percutaneous coronary intervention (PCI). This divergence in findings from those of randomized trials calls into question the generalizability of the randomized trial results to unselected populations and suggests that the selection criteria used in these trials are an important determinant of PCI performance. The periods encompassed by each of these studies, however, predate both thorough understanding of appropriate patient selection and numerous improvements in stent design and in antithrombotic and antiatherosclerotic medical therapy.

To examine the impact on survival of first-time revascularization with PCI with coronary stenting instead of elective CABG, we examined long-term mortality among 


\section{Abbreviations and Acronyms \\ BARI = Bypass Angioplasty Revascularization Investigation \\ $\mathrm{CABG}=$ coronary artery bypass grafting \\ $\mathrm{CAD}=$ coronary artery disease \\ CI $=$ confidence interval \\ HR $\quad=$ hazard ratio \\ MI $=$ myocardial infarction \\ $\mathrm{OR} \quad=$ odds ratio \\ PCI = percutaneous coronary intervention \\ THIRDBase $=$ Texas Heart Institute Research Database}

patients with multivessel CAD enrolled in the Texas Heart Institute Research Database (THIRDBase). THIRDBase is a comprehensive, longitudinal clinical registry of outcomes for more than 150,000 patients treated for cardiovascular disease at the Texas Heart Institute at St Luke's Episcopal Hospital. It includes a wide range of data for all patients admitted to our institution with a diagnosis of cardiovascular disease. Standard methods of survival comparison (Cox proportional hazards modeling and propensity-weighted scoring) and a more stringent statistical method (propensity-based case matching) were used to make our results comparable with those of previous studies.

\section{Materials and Methods \\ Study Population}

All patients included in the study were residents of Texas with multivessel CAD who underwent an isolated first revascularization procedure by either coronary stenting or CABG at the Texas Heart Institute between January 1995 and December 2003. Patients were excluded if they had previous $\mathrm{CABG}$, also required a valve operation, were undergoing primary PCI for an acute myocardial infarction $[\mathrm{MI}]$, had left main $\mathrm{CAD}$, or had a limited life expectancy at the time of revascularization because of malignancy. Baseline data on patient characteristics were obtained prospectively for both groups. Coronary anatomic and procedural characteristics, in-hospital outcomes, and vital status as of December 31, 2004, were collected for all patients. Survivorship was determined from the United States Department of Vital Statistics Database, and followup was complete for $100 \%$ of patients. Our long-term survival analyses included both short- and long-term survival data (perioperative and long-term survivals were not considered separately).

Written, informed consent was obtained at hospital admission from all patients registered in THIRDBase. All data we analyzed were stripped of personal identifiers. We obtained approval for this project from the Committee for the Protection of Human Subjects, the institutional review board for the University of Texas Health Science Center at Houston, on November 11, 2004 (HSCSHIS-04-009).

Patient histories were obtained by interview at hospital or clinic presentation and were entered prospectively into the database. Variables defined were the number of diseased vessels, left ventricular ejection fraction, urgency of the procedure, hyper- tension (defined as blood $>130 / 90 \mathrm{~mm} \mathrm{Hg}$ or current use of antihypertensive medications), angina severity (defined according to the Canadian Cardiovascular Society classification system), congestive heart failure severity (classified according to New York Heart Association criteria), family history of CAD, previous MI, renal insufficiency (defined as a serum creatinine level $\geq 2 \mathrm{mg}$ / $\mathrm{dL}$ ), diabetes mellitus (defined as a fasting blood sugar level $>125 \mathrm{mg} / \mathrm{dL}$ or the use of antidiabetic agents), peripheral vascular disease, transient ischemic attack, cerebrovascular disease, abdominal aortic aneurysm, and chronic obstructive pulmonary disease.

\section{Statistical Analysis}

The Pearson $\chi^{2}$ test was used to analyze discrete variables, and the Student $t$ test was used to analyze continuous variables. Logistic regression and Cox proportional hazard models that used a forward stepwise variable selection process were developed to determine which clinical and angiographic variables were associated with late mortality. This HR (or odds ratio [OR]) is the ratio of the mortality in the stenting group to that in the CABG group at any given point in time, controlling for differences in patient demographic characteristics or risk factors.

To express survival differences in percentages, long-term mortality was evaluated with Cox proportional hazards modeling, with propensity scoring to adjust for differences in baseline characteristics influencing each patient's likelihood of being treated with stenting versus $\mathrm{CABG}$, and with an analysis that involved stringent case matching according to propensity scores. Propensity scores were computed from as many patient characteristics and early outcome variables as possible. For the case-matched study population, pairs of patients with similar propensity scores were selected from the two treatment groups. This method yielded very similar stenting $(\mathrm{n}=1856)$ and CABG $(\mathrm{n}=1856)$ groups, which were compared in subsequent late-outcome analyses. Analyses were performed with SAS 6.09 software (SAS Institute, Inc, Cary, NC) for the VAX/ VMS operating system.

\section{Results}

\section{Patient Characteristics}

Between 1995 and 2004, a total of 6847 patients with multivessel CAD (CABG $n=3917$, stenting $n=2930$ ) who underwent an isolated first revascularization procedure and who were enrolled in the THIRDBase were included in this study. Follow-up extended up to 9 years, with median durations of 3.0 years (25th-75th interquartile range 2.0-7.4 years) for the stenting group and 3.9 years $(25$ th -75 th interquartile range 1.1-5.0 years) for the CABG group.

The prevalences of diabetes (33\%) and reduced left ventricular systolic function (ejection fraction $<50 \%, 32 \%$ ) were high in this study population, particularly in the CABG group (Table 1). The CABG group also had higher prevalences of advanced age, male sex, smoking, previous MI, unstable angina, congestive heart failure, peripheral vascular disease, cerebrovascular accident, and chronic obstructive pulmonary disease. Of the listed variables, only hypercholesterolemia, obesity, and female sex were more 
TABLE 1. Patient data according to revascularization method

\begin{tabular}{|c|c|c|c|}
\hline & CABG $(n=2634)$ & Stenting ( $\mathrm{n}=3845)$ & $P$ value \\
\hline \multicolumn{4}{|l|}{ Demographic data } \\
\hline Mean age (y) & $62.5 \%$ & $61.7 \%$ & .015 \\
\hline Age $>65 y$ & $45.1 \%$ & $43.7 \%$ & .23 \\
\hline Female & $26.0 \%$ & $27.1 \%$ & .29 \\
\hline \multicolumn{4}{|l|}{ Risk factors } \\
\hline Diabetes mellitus & $37.6 \%$ & $29.4 \%$ & $<.001$ \\
\hline Insulin requirement & $12.0 \%$ & $7.4 \%$ & .001 \\
\hline Hypertension & $74.8 \%$ & $75.5 \%$ & .59 \\
\hline Hypercholesterolemia & $58.4 \%$ & $66.2 \%$ & $<.001$ \\
\hline Smoking & $49.8 \%$ & $47.8 \%$ & .11 \\
\hline Obesity (body mass index $>26 \mathrm{~kg} / \mathrm{m}^{2}$ ) & $19.6 \%$ & $21.2 \%$ & .11 \\
\hline \multicolumn{4}{|l|}{ Other medical history data } \\
\hline Family history of coronary artery disease & $36.2 \%$ & $38.3 \%$ & .075 \\
\hline History of myocardial infarction & $34.1 \%$ & $29.5 \%$ & $<.001$ \\
\hline History of unstable angina & $54.2 \%$ & $52.1 \%$ & .079 \\
\hline History of congestive heart failure & $13.9 \%$ & $11.7 \%$ & .009 \\
\hline New York Heart Association functional class IV & $11.1 \%$ & $10.4 \%$ & .38 \\
\hline History of nonsurgical valve disease & $4.8 \%$ & $5.5 \%$ & .16 \\
\hline History of peripheral vascular disease & $17.8 \%$ & $15.8 \%$ & .025 \\
\hline History of chronic renal insufficiency & $12.2 \%$ & $12.2 \%$ & .99 \\
\hline Previous cerebrovascular accident & $5.9 \%$ & $5.0 \%$ & .097 \\
\hline History of chronic obstructive pulmonary disease & $22.9 \%$ & $19.6 \%$ & .001 \\
\hline \multicolumn{4}{|l|}{ Angiographic data } \\
\hline Disease type and extent & & & $<.001$ \\
\hline Two-vessel disease & $23.1 \%$ & $72.8 \%$ & \\
\hline Three-vessel disease & $73.5 \%$ & $27.2 \%$ & \\
\hline Left ventricular function & & & $<.001$ \\
\hline Ejection fraction $\geq 50 \%$ & $64.0 \%$ & $71.1 \%$ & \\
\hline Ejection fraction $35 \%-49 \%$ & $24.6 \%$ & $20.8 \%$ & \\
\hline Ejection fraction $\leq 34 \%$ & $11.3 \%$ & $8.1 \%$ & \\
\hline Urgent procedure & $5.7 \%$ & $4.9 \%$ & .16 \\
\hline
\end{tabular}

$C A B G$, Coronary artery bypass grafting.

common in the stenting group. Most patients in the CABG group $(76 \%)$ had three-vessel CAD, whereas those in the stenting group had a more variable degree of CAD: $72 \%$ had two-vessel CAD, and 27\% had three-vessel CAD. Urgent procedures accounted for $5.3 \%$ of all cases $(5.7 \%$ for CABG, $4.9 \%$ for stenting, $P=.1571$ ).

Patients in the CABG group each had an average of 3.4 distal anastomoses performed. A left internal thoracic graft to the left anterior descending coronary artery was used in 93\% of patients. In the stenting group, glycoprotein IIb and IIIa inhibitors were used in $18 \%$ of stenting procedures.

\section{Predictors}

Several significant predictors of mortality were identified (Table 2). The multivariable logistic regression model showed that the strongest predictors of mortality in the CABG group were age older than 65 years (OR 2.2, 95\% confidence interval [CI] 1.8-2.7) and history of chronic renal insufficiency (OR 1.9, 95\% CI 1.5-2.3). The significant predictors of mortality in the stenting group included age older than 65 years (OR 2.8, 95\% CI 2.1-3.8) and history of chronic renal insufficiency (OR 2.2, 95\% CI 1.6-3.1).

\section{Short-term Survival Analysis}

Adjusted in-hospital mortality was higher in the CABG group than in the stenting group $(2.0 \%$ vs $0.3 \%, P<$ .0001). Risk of in-hospital death was also higher in the CABG group (HR 2.9, 95\% CI 1.7-4.7). Additionally, CABG was associated with greater risks of Q-wave MI (3.6\% vs $1.0 \%, P<.0001)$ and periprocedural major stroke $(2.5 \%$ vs $0.2 \% ; P<.0001)$ than was stenting. A higher rate of repeat revascularization during initial hospitalization was noted in the stenting group $(0.1 \%$ vs $2.5 \%, P<.0001)$. The rates of the composite in-hospital end point of death, MI, stroke, and repeat revascularization were $7.3 \%$ in the $\mathrm{CABG}$ group and $4.0 \%$ in the stenting group $(P<.0001)$.

\section{Long-term Mortality}

Unadjusted long-term mortality was similar between the $\mathrm{CABG}$ group and the stenting group (OR 1.1, 95\% CI 
TABLE 2. Variables associated with long-term mortality in Cox regression analysis

\begin{tabular}{|c|c|c|c|c|}
\hline & \multicolumn{2}{|r|}{ CABG } & \multicolumn{2}{|r|}{ Stenting } \\
\hline & Odds ratio & 95\% Confidence interval & Odds ratio & 95\% Confidence interva \\
\hline Age $>65$ y & 2.2 & $1.8-2.7$ & 2.8 & $2.1-3.8$ \\
\hline New York Heart Association functional class IV & 1.5 & $1.1-2.0$ & NS & \\
\hline History of valve disease not treated surgically & 1.6 & $1.1-2.0$ & NS & \\
\hline History of chronic obstructive pulmonary disease & 1.5 & $1.2-1.8$ & NS & \\
\hline Previous cerebrovascular accident & 1.6 & $1.2-2.2$ & NS & \\
\hline History of peripheral vascular disease & 1.7 & $1.4-2.1$ & NS & \\
\hline History of chronic renal insufficiency & 1.9 & $1.5-2.3$ & 2.2 & $1.6-3.1$ \\
\hline History of congestive heart failure & 1.8 & $1.4-2.3$ & 2.1 & $1.3-3.4$ \\
\hline Diabetes mellitus & 1.6 & $1.3-2.1$ & 1.7 & $1.1-2.6$ \\
\hline
\end{tabular}

$C A B G$, Coronary artery bypass grafting; $N S$, not significant.

1.0-1.2, log-rank test $P=.27$ ). After adjustment with a multivariate model, no difference in mortality was observed between the CABG and stenting groups (OR 0.9, 95\% CI 0.7-1.1). Propensity adjustment, however, revealed a rather strongly favorable result for surgically treated patients (HR $0.8,95 \%$ CI $0.7-0.9, P=.0005)$.

\section{Matched Groups}

We matched 3712 patients (1856 from each group) with respect to their likelihood of being assigned to undergo CABG or stenting according to their clinical, angiographic, and demographic characteristics (Table 3). Between these two groups, there were no significant differences in age, sex distribution, or the prevalences of current smoking, diabetes, hypercholesterolemia, presentation with unstable angina, or history of MI. The angiographic characteristics of both groups of patients were also similar; both groups had large proportions of patients with two-vessel CAD. The average number of totally occlusive lesions was 1.2 in the $\mathrm{CABG}$ group and 1.4 in the stenting group $(P=.049)$. Similar to the outcome of adjusting observed values, the matched population without adjustment in observed values revealed a significant difference in favor of surgery at 9 years (HR $0.7,95 \%$ CI 0.6-0.9, $P=.004)$.

TABLE 3. Patient data by revascularization procedure in case-matched patients

\begin{tabular}{|c|c|c|c|}
\hline & CABG $(n=1856)$ & Stenting $(\mathrm{n}=1856)$ & $P$ value \\
\hline \multicolumn{4}{|l|}{ Demographic data } \\
\hline Age $>65$ y & $41.0 \%$ & $42.0 \%$ & .54 \\
\hline Female & $28.9 \%$ & $27.7 \%$ & .41 \\
\hline \multicolumn{4}{|l|}{ Risk factors } \\
\hline Any diabetes mellitus & $28.9 \%$ & $28.8 \%$ & .94 \\
\hline Hypertension & $72.1 \%$ & $71.7 \%$ & .78 \\
\hline Hypercholesterolemia & $65.0 \%$ & $62.8 \%$ & .17 \\
\hline Smoking & $50.3 \%$ & $49.5 \%$ & 62 \\
\hline Obesity (body mass index $>26 \mathrm{~kg} / \mathrm{m}^{2}$ ) & $19.5 \%$ & $19.3 \%$ & .86 \\
\hline \multicolumn{4}{|l|}{ Other medical history data } \\
\hline Family history of coronary artery disease & $38.3 \%$ & $40.0 \%$ & .30 \\
\hline History of myocardial infarction & $10.3 \%$ & $11.3 \%$ & .33 \\
\hline History of unstable angina & $58.4 \%$ & $55.9 \%$ & .12 \\
\hline History of congestive heart failure & $12.1 \%$ & $11.7 \%$ & .75 \\
\hline New York Heart Association functional class III or IV & $68.6 \%$ & $68.8 \%$ & .91 \\
\hline History of valve disease & $4.6 \%$ & $3.9 \%$ & .31 \\
\hline History of peripheral vascular disease & $15.3 \%$ & $14.3 \%$ & .42 \\
\hline History of chronic renal insufficiency & $12.1 \%$ & $10.1 \%$ & .06 \\
\hline Previous cerebrovascular accident & $4.2 \%$ & $4 \%$ & .67 \\
\hline History of chronic obstructive pulmonary disease & $20.2 \%$ & $19.4 \%$ & .58 \\
\hline Urgent procedure & $5.7 \%$ & $4.7 \%$ & .22 \\
\hline Totally occlusive lesions per patient (mean no.) & 1.2 & 1.4 & .049 \\
\hline
\end{tabular}

$C A B G$, Coronary artery bypass grafting. 
TABLE 4. Large-scale observational studies of percutaneous coronary intervention versus coronary artery bypass grafting with long-term follow-up

\begin{tabular}{|c|c|c|c|c|c|c|c|c|c|c|}
\hline & \multicolumn{2}{|c|}{$\begin{array}{c}\text { Thoraxcenter, }^{7} \\
\text { 1995-1999 }\end{array}$} & \multicolumn{2}{|c|}{$\begin{array}{c}\text { Cleveland } \\
\text { Clinic, }{ }^{1} \text { 1995-1999 }\end{array}$} & \multicolumn{2}{|c|}{$\begin{array}{c}\text { New York } \\
\text { State, }{ }^{2} \text { 1997-2000 } \\
\end{array}$} & \multicolumn{2}{|c|}{$\begin{array}{c}\text { Northern New } \\
\text { England, }{ }^{6} \text { 1994-2001 }\end{array}$} & \multicolumn{2}{|c|}{$\begin{array}{c}\text { Current study, } \\
\text { 1995-2004 }\end{array}$} \\
\hline & CABG & PCI & CABG & PCI & CABG & PCI & CABG & PCI & CABG & PCI \\
\hline Mean follow-up (y) & 7 & & 5.2 & & 1.9 & 1.6 & 3.6 & & 3.5 & \\
\hline No. of patients & 409 & 1640 & 5161 & 872 & 22,102 & 37,212 & 4295 & 10,198 & 2930 & 3917 \\
\hline Received stents & NA & $100 \%$ & NA & $70 \%$ & NA & $100 \%$ & NA & $64 \%$ & NA & $100 \%$ \\
\hline Mean age (y) & 63 & 61 & 64 & 65 & 67 & 65 & 64 & 62 & 63 & 62 \\
\hline Diabetes mellitus & $15 \%$ & $23 \%$ & $40 \%$ & $30 \%$ & $33 \%$ & $25 \%$ & $34 \%$ & $26 \%$ & $37 \%$ & $30 \%$ \\
\hline $\begin{array}{l}\text { History of chronic } \\
\text { renal insufficiency }\end{array}$ & - & - & $3 \%$ & $6 \%$ & $3 \%$ & $6 \%$ & $3 \%$ & $2 \%$ & $12 \%$ & $12 \%$ \\
\hline Three-vessel disease & $80 \%$ & $23 \%$ & $74 \%$ & $33 \%$ & $69 \%$ & $19 \%$ & $50 \%$ & $13 \%$ & $73 \%$ & $27 \%$ \\
\hline $\begin{array}{l}\text { Depressed }(<50 \%) \text { left } \\
\text { ventricular EF }\end{array}$ & $23 \%$ & $13 \%$ & Mea & $54 \%$ & NR & $19 \%$ & $14 \%$ & $6 \%$ & $36 \%$ & $29 \%$ \\
\hline \multicolumn{11}{|l|}{ Mortality } \\
\hline $30-d$ & $0.2 \%$ & $1.0 \%$ & $1.3 \%$ & $1.1 \%$ & $1.8 \%$ & $0.7 \%$ & $1.7 \% \dagger$ & $0.5 \% \dagger$ & $1.4 \% \dagger$ & $0.3 \% \dagger$ \\
\hline Long-term $\ddagger$ & \multicolumn{2}{|c|}{$0.59(0.38-0.9)$} & \multicolumn{2}{|c|}{$0.43(0.35-0.54)$} & \multicolumn{2}{|c|}{$0.64-0.75$} & \multicolumn{2}{|c|}{$0.86(0.77-0.97)$} & \multicolumn{2}{|c|}{$0.8(0.7-0.9)$} \\
\hline
\end{tabular}

$C A B G$, Coronary artery bypass grafting; $P C l$, percutaneous coronary intervention; $N A$, not applicable; $E F$, ejection fraction; $N R$, not reported. *Excluded by design. †Risk-adjusted 30 -day mortality. ł̦Long-term mortality data are reported as hazard ratios.

\section{Discussion}

The patients included in this study represent a broad population of patients with CAD of varied clinical presentation who would be candidates for either CABG or stenting in the current era. Reflecting current clinical practice, $\mathrm{CABG}$ was generally reserved for patients with higher-risk profiles, more lesions, and worse left ventricular systolic function. The results of our survival analysis, stringently adjusted for these confounding factors-including number of diseased vessels—suggest that, for primary revascularization at least, performing $C A B G$ rather than PCI with stenting carries a greater short-term cost in terms of complications and mortality but provides more effective protection and improved survival in the long term.

Our findings have variable companionship in the literature. The Bypass Angioplasty Revascularization Investigation (BARI) found poorer 7-year survival after angioplasty $(65 \%)$ than after CABG $(80 \%)$ in patients being treated for diabetes. ${ }^{3}$ A separate analysis of BARI registry data ${ }^{4}$ disagreed with those of randomized trial data with respect to the outcomes of patients with diabetes: patients with diabetes treated percutaneously and those treated surgically had identical survival rates $(84 \%)$ during 7 years. A review of the characteristics of the percutaneously treated patients in the BARI trial and registry ${ }^{5}$ found that registry patients treated percutaneously had fewer lesions at baseline and that their lesion characteristics were more favorable for PCI. These observations highlight the impact of patient selection on longterm outcome after a PCI procedure.

During the performance and follow-up of several randomized trials comparing angioplasty with $\mathrm{CABG}$, the coronary stent was introduced and rapidly became a standard of care. Many clinicians assume that stenting is more beneficial for long-term survival than is angioplasty alone, but this assumption has not been tested in a randomized trial. Our data suggest that in an unselected population of patients with multivessel CAD first revascularization with CABG is associated with better survival rates than those associated with coronary stenting. These results are in agreement with those of similarly designed large-scale retrospective studies, despite differences in study design and population (Table 4). In the Cleveland Clinic, ${ }^{1}$ New York State Registries, ${ }^{2}$ Northern New England, ${ }^{6}$ and Thoraxcenter ${ }^{7}$ database analyses, CABG was associated with lower long-term mortality than was stenting.

On the other hand, our findings contradict those of the Stent or Surgery trial (SoS) ${ }^{8}$ ERACI II, ${ }^{9}$, and Randomized Intervention Treatment of Angina (RITA) ${ }^{10}$ randomized trials. Like BARI and the trials of balloon angioplasty without stenting, these three studies found no difference in 5-year survival between patients treated with stenting or CABG. Also like BARI, these studies found that patients with diabetes may fare slightly less well when treated percutaneously. In our study, the improved survivals in the CABG group in both the risk-adjusted analyses and the case-matched comparison suggests that our findings were not merely the result of an unequal distribution of risk factors. An intriguing potential explanation for the difference between our results and those of other randomized trials is the inclusion in our study of patients with treatment similar to current practice patterns. After the publication of the BARI data and the accumulation of experience with long-term stent failure by 
clinicians, physician acceptance of these data was somewhat heterogeneous. Our institution's increasing tendency during recent years to use PCI (rather than surgical) therapy in patients with complex or bifurcation lesions and in patients with diabetes and severe CAD may be responsible for the differences between our results and those of randomized controlled trials in terms of the outcomes of the two treatment strategies.

Moreover, CAD progression during follow-up is a potential adverse outcome of both techniques. ${ }^{11}$ ). Medical therapy, including the routine use of angiotensin-converting enzyme inhibitors, long-term dual antiplatelet therapy, and far more aggressive lipid-lowering therapy, has evolved significantly since the previous registry studies were published. Both saphenous vein grafts and native vessels receive the protection of more aggressive medical therapy. Still, CAD progression does occur. Failure of a percutaneously treated vessel because of lesion progression at an untreated site may have greater impact on the subtended tissue than does lesion progression after $\mathrm{CABG}$, in which two or more vessels may share the responsibility (albeit unevenly). As a result, patients who undergo $\mathrm{CABG}$ may receive greater relative benefit from these medical innovations while being less susceptible to catastrophe in the case of CAD progression than those who undergo PCI. Attempts to treat percutaneously patients or vessels in which eventual treatment failure is probable (eg, patients with diabetes mellitus, extensive atherosclerotic burden, small vessels with long lesions, or bifurcations) may highlight this contrast between the two revascularization methods.

Hannan and colleagues ${ }^{2}$ used the powerful New York State revascularization reporting system to examine outcomes in more than 59,000 patients treated between 1997 and 2000. Patients with previous revascularization procedures and left main coronary artery stenosis were excluded. Survival after 3 years was better in the stenting group than in the CABG group. After adjustment for baseline risk factors, however, CABG was associated with superior survival not only in the population as a whole but, surprisingly, in almost every subgroup examined, including patients with twovessel CAD without left anterior descending coronary artery involvement.

Brener and associates ${ }^{1}$ examined the survivals of patients treated surgically or percutaneously at the Cleveland Clinic from 1995 to 1999, excluding patients who had been refused treatment by a cardiac surgeon because of "comorbidity or a lack of appropriate target vessels" and patients who were undergoing primary treatment for acute MI, who died during the procedure, or who had no social security number. Only $70 \%$ of the percutaneously treated patients underwent stent implantation. After 5 years, survivals were similar in patients who underwent PCI $(16 \%)$ and those who underwent CABG (14\%), despite the fact that the CABG group had more risk factors at baseline. After propensity matching was used to correct for this difference in baseline risk, the CABG group appeared to have superior survival (OR 2.3, 95\% CI 1.92.9). We also excluded patients with acute MI in this study, but we included patients with any other high-risk surgical profile, and our PCI population was restricted to those who received stents.

The Thoraxcenter study ${ }^{7}$ examined a population similar in size and characteristics to that of the Cleveland Clinic study, save that only patients who received a stent in two or more vessels were included. The finding that 8-year survival was marginally better in the CABG group $(87.5 \%)$ than in the stenting group $(82 \%)(P=.06)$ was driven almost solely by the inclusion of patients with left main CAD.

Additionally, the Cleveland Clinic ${ }^{1}$ and Northern New England ${ }^{6}$ studies included patients treated with balloon angioplasty only (without stenting). In contrast, the New York State Registries ${ }^{2}$ and Thoraxcenter ${ }^{7}$ trials restricted their PCI populations to stent recipients; however, these two studies differed substantially in the demographic characteristics of their populations.

\section{Limitations}

Even large observational studies such as this one can be biased by baseline differences between treatment groups. The main limitation of this study is that although the observations that prompted physicians to refer patients for coronary revascularization may be important markers of outcome, we could not obtain these data. We have attempted to nullify the effects of these data and other potential confounding variables by using Cox and propensity analysis. The differences in the results of the Cox and propensity analyses are a source of interest. Although it is possible that propensity adjustment overcorrects for differences in baseline variables, this seems unlikely given the results seen in the matched populations. A more probable explanation is that the ability of the proportional hazards model to separate the effects of individual variables may exclude the effect of physician judgment. Propensity adjustment or matching by examining the confluence of variables affecting referral for a given procedure may be a more powerful means of statistical adjustment for a study such as this.

Long-term follow-up data on the need for repeat procedures or control of angina were not available. It is well established, however, that CABG is more reliable than bare-metal stenting in preventing symptom recurrence and the need for repeat procedures. Our goal was to examine the effect of that difference on long-term survival in a nonrandomized population.

A limitation of both our study and all other comparative studies of CABG and PCI is that their findings may quickly be outdated by technologic advances. One such advance is the advent of drug-eluting stents, which dramatically reduce the need for repeat revascularization after stent placement. There is not yet clear-cut evidence, however, that these stents 
improve survival. We therefore may still draw inferences about comparative survival from populations with bare-metal stents. Additionally, uses of adjunct devices, such as cutting balloon, laser, and rotational atherectomy, although rare in our population, were not recorded, so the influences of such devices on outcome are not known. Further, advances in preoperative evaluation, including more precise coronary artery and myocardial imaging and diagnostic techniques, have allowed more appropriate patient selection and surgical planning. Improvements in cardiopulmonary perfusion and careful myocardial protection, as well as the use of off-pump and on-pump beating-heart techniques in selected cases, have also decreased perioperative morbidity and mortality.

\section{Implications}

In this large, longitudinal database study performed during the modern era of percutaneous coronary stenting, physician-selected strategies for first-time revascularization with CABG were associated with better long-term survival than was stent-assisted angioplasty.

We thank Stephen N. Palmer, PhD, ELS, who provided editorial support.

\section{References}

1. Brener SJ, Lytle BW, Casserly IP, Schneider JP, Topol EJ, Lauer MS. Propensity analysis of long-term survival after surgical or percutaneous revascularization in patients with multivessel coronary artery disease and high-risk features. Circulation. 2004;109:2290-5.

2. Hannan EL, Racz MJ, Walford G, Jones RH, Ryan TJ, Bennett E, et al. Long-term outcomes of coronary-artery bypass grafting versus stent implantation. N Engl J Med. 2005;352:2174-83.
3. Comparison of coronary bypass surgery with angioplasty in patients with multivessel disease. The Bypass Angioplasty Revascularization Investigation (BARI) Investigators [Published erratum appears in N Engl J Med. 1997;336:147]. N Engl J Med. 1996;335:217-25.

4. Feit F, Brooks MM, Sopko G, Keller NM, Rosen A, Krone R, et al. Long-term clinical outcome in the Bypass Angioplasty Revascularization Investigation Registry: comparison with the randomized trial. BARI Investigators. Circulation. 2000;101:2795-802.

5. Brooks MM, Jones RH, Bach RG, Chaitman BR, Kern MJ, Orszulak TA, et al. Predictors of mortality and mortality from cardiac causes in the bypass angioplasty revascularization investigation (BARI) randomized trial and registry. For the BARI Investigators. Circulation. 2000;101:2682-9.

6. Malenka DJ, Leavitt BJ, Hearne MJ, Robb JF, Baribeau YR, Ryan TJ, et al. Comparing long-term survival of patients with multivessel coronary disease after CABG or PCI: analysis of BARI-like patients in northern New England. Circulation. 2005;112(9 Suppl):I371-6.

7. van Domburg RT, Takkenberg JJ, Noordzij LJ, Saia F, van Herwerden LA, Serruys PW, et al. Late outcome after stenting or coronary artery bypass surgery for the treatment of multivessel disease: a single-center matched-propensity controlled cohort study. Ann Thorac Surg. 2005;79:1563-9.

8. SoS Investigators. Coronary artery bypass surgery versus percutaneous coronary intervention with stent implantation in patients with multivessel coronary artery disease (the Stent or Surgery trial): a randomised controlled trial. Lancet. 2002;360:965-70.

9. Rodriguez A, Bernardi V, Navia J, Baldi J, Grinfeld L, Martinez J, et al. Argentine Randomized Study: Coronary Angioplasty with Stenting versus Coronary Bypass Surgery in patients with Multiple-Vessel Disease (ERACI II): 30-day and one-year follow-up results. ERACI II Investigators. J Am Coll Cardiol. 2001;37:51-8.

10. Coronary angioplasty versus coronary artery bypass surgery: the Randomized Intervention Treatment of Angina (RITA) trial. Lancet. 1993; 341:573-80.

11. Alderman EL, Kip KE, Whitlow PL, Bashore T, Fortin D, Bourassa MG, et al. Native coronary disease progression exceeds failed revascularization as cause of angina after five years in the Bypass Angioplasty Revascularization Investigation (BARI). J Am Coll Cardiol. 2004;44:766-74. 